\title{
TITLE:
}

\section{Hartle-Hawking state is a maximum of entanglement entropy}

$\operatorname{AUTHOR}(S)$ :

Mukohyama, S

CITATION:

Mukohyama, S. Hartle-Hawking state is a maximum of entanglement entropy. PHYSICAL REVIEW D 2000, 61(6): 064015.

ISSUE DATE:

2000-03-15

URL:

http://hdl.handle.net/2433/50456

RIGHT:

Copyright 2000 American Physical Society 
PHYSICAL REVIEW D, VOLUME 61, 064015

\title{
Hartle-Hawking state is a maximum of entanglement entropy
}

\author{
Shinji Mukohyama \\ Yukawa Institute for Theoretical Physics, Kyoto University, Kyoto 606-8502, Japan \\ and Department of Physics and Astronomy, University of Victoria, Victoria BC, Canada V8W 3P6
}

(Received 2 April 1999; published 24 February 2000)

\begin{abstract}
It is shown that the Hartle-Hawking state of a scalar field is a maximum of entanglement entropy in the space of pure quantum states satisfying the condition that the back reaction be finite. In other words, the Hartle-Hawking state is a curved-space analogue of the EPR state, which is also a maximum of entanglement entropy.
\end{abstract}

PACS number(s): 04.70.Dy

Entanglement entropy $[1,2]$ is one of the strongest candidates for the origin of black hole entropy [3,4]. It originates from a direct-sum structure of a Hilbert space of a quantum system: for an element $|\psi\rangle$ of the Hilbert space $\mathcal{F}$ of the form

$$
\mathcal{F}=\mathcal{F}_{I} \bar{\otimes} \mathcal{F}_{I I},
$$

the entanglement entropy $S_{\text {ent }}$ is defined by

$$
\begin{gathered}
S_{e n t}=-\operatorname{Tr}_{I}\left[\rho_{I} \ln \rho_{I}\right], \\
\rho_{I}=\operatorname{Tr}_{I I}|\psi\rangle\langle\psi| .
\end{gathered}
$$

Here $\bar{\otimes}$ denotes a tensor product followed by a suitable completion and $\operatorname{Tr}_{I, I I}$ denotes a partial trace over $\mathcal{F}_{I, I I}$, respectively.

In Ref. [5], a new interpretation of the entanglement entropy was proposed based on its relation to the so-called conditional entropy and a well-known meaning of the latter. It was conjectured that the entanglement entropy for a pure state is an amount of information, which can be transmitted through $\mathcal{F}_{I I}$ and $\mathcal{F}_{I}$ from a system interacting with $\mathcal{F}_{I I}$ to another system interacting with $\mathcal{F}_{I}$ by using quantum entanglement. Thus, it is important to seek quantum states having maximal entanglement entropy and to investigate properties of the states. In fact, it was shown that a state having maximal entanglement entropy plays an important role in quantum teleportation [5].

In this paper, we show that the Hartle-Hawking state [6] of a scalar field is a maximum of entanglement entropy in the space of pure states satisfying a consistency condition.

For simplicity, we consider a minimally coupled, real scalar field described by the action

$$
S=-\frac{1}{2} \int d^{4} x \sqrt{-g}\left[g^{\mu \nu} \partial_{\mu} \phi \partial_{\nu} \phi+m^{2} \phi^{2}\right]
$$

in the spherically symmetric, static black-hole spacetime

$$
d s^{2}=-f(r) d t^{2}+\frac{d r^{2}}{f(r)}+r^{2} d \Omega^{2}
$$

We denote the area radius of the horizon by $r_{0}$ and the surface gravity by $\kappa_{0}(\neq 0)$ :

$$
f\left(r_{0}\right)=0,
$$

$$
\kappa_{0}=\frac{1}{2} f^{\prime}\left(r_{0}\right)
$$

We quantize the system of the scalar field with respect to the Killing time $t$ in a Kruskal-like extension of the black hole spacetime. The corresponding ground state is called the Boulware state and its energy density is known to diverge near the horizon. Although we shall only consider states with bounded energy density, it is convenient to express these states as excited states above the Boulware ground state for technical reasons. Hence, we would like to introduce an ultraviolet cutoff $\alpha$ with dimension of length to control the divergence. Off course, we shall take the limit $\alpha \rightarrow 0$ in the end. The cutoff parameter $\alpha$ is implemented so that we only consider two regions satisfying $r>r_{1}$ (shaded regions I and II in Fig. 1), where $r_{1}\left(>r_{0}\right)$ is determined by

$$
\alpha=\int_{r_{0}}^{r_{1}} \frac{d r}{\sqrt{f(r)}} .
$$

(Strictly speaking, we also have to introduce outer boundaries, say at $r=L\left(\gg r_{0}\right)$, to control the infinite volume of the constant- $t$ surface. However, even if there are outer boundaries, the following arguments still hold and we can take the limit $L \rightarrow \infty$ in the end.) Evidently, the limit $\alpha \rightarrow 0$ corresponds to the limit $r_{1} \rightarrow r_{0}$. Thus, in this limit, the whole region in which $\partial / \partial t$ is timelike is considered.

In this situation, there is a natural choice for division of the system of the scalar field: let $\mathcal{H}_{I}$ be the space of mode functions with supports in the region I and $\mathcal{H}_{I I}$ be the space of mode functions with supports in the region II. Thence, the

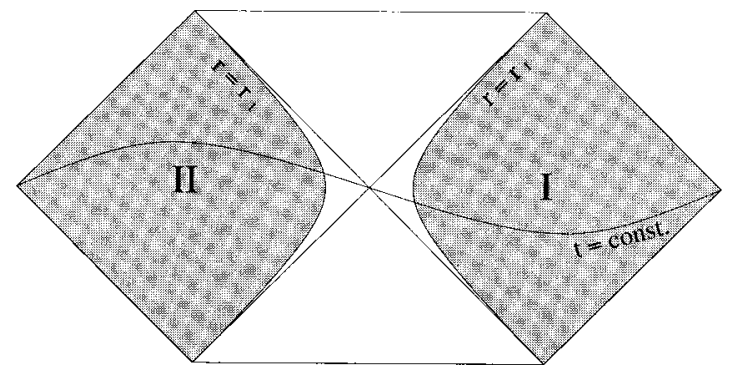

FIG. 1. The Kruskal-like extension of the static, spherically symmetric black-hole spacetime. We consider only the regions satisfying $r>r_{1}$ (the shaded regions I and II). 
space $\mathcal{F}$ of all states are of the form (1), where $\mathcal{F}_{I}$ and $\mathcal{F}_{I I}$ are defined as symmetric Fock spaces constructed from $\mathcal{H}_{I}$ and $\mathcal{H}_{\text {II }}$, respectively:

$$
\begin{gathered}
\mathcal{F}_{I} \equiv C \oplus \mathcal{H}_{I} \oplus\left(\mathcal{H}_{I} \bar{\otimes} \mathcal{H}_{I}\right)_{s y m} \oplus \cdots, \\
\mathcal{F}_{I I} \equiv C \oplus \mathcal{H}_{I I} \oplus\left(\mathcal{H}_{I I} \bar{\otimes} \mathcal{H}_{I I}\right)_{s y m} \oplus \cdots
\end{gathered}
$$

Here $(\cdots)_{\text {sym }}$ denotes the symmetrization.

Let us investigate what kind of condition should be imposed for our arguments to be self-consistent. A clear condition is that the back reaction of the scalar field to the background geometry should be finite. Thus, the contribution $\Delta M$ of the subsystem $\mathcal{F}_{I}$ to the mass of the background geometry should be bounded in the limit $\alpha \rightarrow 0$. Although this condition is not a sufficient condition in order to make the back reaction finite, what is surprising is that we can obtain the HartleHawking state without using any other conditions stronger than this. In this sense, our strategy of maximizing entanglement entropy seems as strong as the maximum entropy principle in the statistical thermodynamics.

As shown in Ref. [7], $\Delta M$ is given by

$$
\Delta M \equiv-\int_{x \in I} T_{t}^{t} 4 \pi r^{2} d r=H_{I}
$$

where $H_{I}$ is the Hamiltonian of the subsystem $\mathcal{F}_{I}$ with respect to the Killing time $t$. Hence, the expectation value of $\Delta M$ with respect to a state $|\psi\rangle$ of the scalar field is decomposed into the contribution of excitations and the contribution from the zero-point energy:

$$
\langle\psi|\Delta M| \psi\rangle=E_{e n t}+\Delta M_{B},
$$

where $E_{\text {ent }}$ is the entanglement energy ${ }^{1}$ defined by

$$
E_{e n t} \equiv\left\langle\psi\left|: H_{I}:\right| \psi\right\rangle,
$$

and $\Delta M_{B}$ is the zero-point energy of the Boulware state. Here, the colons denote the usual normal ordering.

Since the Boulware energy $\Delta M_{B}$ diverges as $\Delta M_{B} \simeq$ $-c A T_{H} \alpha^{-2}$ in the limit $\alpha \rightarrow 0$, we should impose the condition

$$
E_{\text {ent }} \simeq\left|\Delta M_{B}\right|
$$

in the lowest order in $\alpha$, where $A=4 \pi r_{0}^{2}$ is the area of the horizon, $T_{H}=\kappa_{0} / 2 \pi$ is the Hawking temperature and $c$ is a positive constant of order unity. We call this condition the small back reaction condition $(S B C) .^{2}$

\footnotetext{
${ }^{1}$ This definition corresponds to $E_{\text {ent }}^{\left(I^{\prime}\right)}$ in Ref. [8] and $\left\langle: H_{2}:\right\rangle$ in Ref. [9].

${ }^{2}$ Off course, finite energy can be added to the entanglement energy without generating a divergent back reaction. However, the finite energy is higher order in the $\alpha$ expansion. Thus, effects of the finite energy are higher order in $\alpha$.
}

Note that the right hand side of SBC (11) is independent of the state $|\psi\rangle$. Thus, SBC implies that the entanglement energy should be fixed when we maximize the entanglement entropy. In statistical thermodynamics, it is well known in what situation we should fix the average energy: we have to fix it when we know the observed value of the energy. However, the corresponding situation seems not to have been essentially known for (quantum or thermal) excitations of fields on a black hole background. In fact, for the brick wall model, in which thermal excitations are considered, Ref. [7] is the first which pointed out that the ground state for the brick wall model is the Boulware state and that the Boulware (negative, divergent) energy should be added to thermal energy. (See Refs. [10-12] for the complete confusion reigning in this subject.) For our system in this paper, it is the Boulware (negative, divergent) energy that makes us fix the entanglement energy.

Now, we shall show that the Hartle-Hawking state is a maximum of the entanglement entropy in the space of pure quantum states satisfying SBC. For this purpose, we prove a more general statement for a quantum system with a state space of the form (1): a state of the form

$$
|\psi\rangle=\mathcal{N} \sum_{n} e^{-E_{n} / 2 T}|n\rangle_{I} \otimes|n\rangle_{I I}
$$

is a maximum of the entanglement entropy in the space of pure states with fixed expectation value of the operator $E_{I}$ defined by

$$
E_{I}=\left(\sum_{n} E_{n}|n\rangle_{I} \cdot{ }_{I}\langle n|\right) \otimes\left(\sum_{m}|m\rangle_{I I} \cdot{ }_{I I}\langle m|\right),
$$

provided that the real constant $T$ is determined so that the expectation value of $E_{I}$ is actually a fixed value. Here, $\mathcal{F}_{I}$ and $\mathcal{F}_{I I}$ are assumed to be isomorphic to each other, $\left\{|n\rangle_{I}\right\}$ and $\left\{|n\rangle_{I I}\right\} \quad(n=1,2, \ldots)$ are bases of the subspaces $\mathcal{F}_{I}$ and $\mathcal{F}_{I I}$, respectively, and $E_{n}$ are assumed to be real and positive. Note that this statement looks almost the same as the following statement in statistical thermodynamics: a canonical state is a maximum of statistical entropy in the space of states with fixed energy, provided that the temperature of the canonical state is determined so that the energy is actually the fixed value. Thus, it might be expected that the above general statement might be directly derived from the standard Jaynes method [13] as this statement in statistical thermodynamics can be derived. However, the Jaynes method cannot be applied directly to our system since the Jaynes method treats entropy of not a subsystem but a total system. Thus, in the proof given below, we seek a correspondence between our variational principle and the standard variational principle in statistical thermodynamics. [See Eq. (23).]

Note that the expectation value of $E_{I}$ is equal to the entanglement energy (10), provided that $|n\rangle_{I}$ and $E_{n}$ are an eigenstate and an eigenvalue of the normal-ordered Hamiltonian : $H_{I}$ : of the subsystem $\mathcal{F}_{I}$. Hence, for the system of the scalar field, the above general statement insists that the state (12) is a maximum of the entanglement entropy in the space of pure states satisfying the SBC, which corresponds 
to fixing the entanglement energy. Of course, in this case, the constant $T$ should be determined so that SBC (11) is satisfied.

Returning to the subject, let us prove the general statement. (The following proof is almost the same as that given in the Appendix of Ref. [5] for a slightly different statement. However, for completeness, we shall give the proof.)

First, we decompose an element $|\psi\rangle$ of $\mathcal{F}$ as

$$
|\psi\rangle=\sum_{n, m} C_{n m}|n\rangle_{I} \otimes|m\rangle_{I I},
$$

where the coefficients $C_{n m}(n, m=1,2, \ldots)$ are complex numbers satisfying $\Sigma_{n, m}\left|C_{n m}\right|^{2}=1$ and can be considered as matrix elements of a matrix $C$. Since $C^{\dagger} C$ is a non-negative Hermitian matrix, it can be diagonalized as

$$
C^{\dagger} C=V^{\dagger} P V
$$

where $P$ is a diagonal matrix with diagonal elements $p_{n}$ $(\geqslant 0)$ and $V$ is a unitary matrix. For this decomposition and diagonalization, the entanglement entropy and the expectation value of the operator $E_{I}$ are written as follows:

$$
\begin{aligned}
& S_{e n t}=-\sum_{n} p_{n} \ln p_{n}, \\
& E_{e n t}=\sum_{n, m} E_{n} p_{m}\left|V_{n m}\right|^{2},
\end{aligned}
$$

where $V_{n m}$ is matrix elements of $V$. The constraints $\sum_{n, m}\left|C_{n m}\right|^{2}=1$ and $V^{\dagger} V=\mathbf{1}$ are equivalent to

$$
\begin{gathered}
\sum_{n} p_{n}=1, \\
\sum_{l} V_{l n}^{*} V_{l m}=\delta_{n m} .
\end{gathered}
$$

Next, we shall show that these expressions are equivalent to those appearing in statistical mechanics in $\mathcal{F}_{I}$. Let us consider a density operator $\bar{\rho}$ on $\mathcal{F}_{I}$ :

$$
\bar{\rho}=\sum_{n, m} \widetilde{P}_{n m}|n\rangle_{I} \cdot{ }_{I}\langle m|,
$$

where $\left(\widetilde{P}_{n m}\right)$ is a non-negative Hermitian matrix with unit trace. By diagonalizing the matrix $\widetilde{P}$ as

$$
\widetilde{P}=\bar{V}^{\dagger} \bar{P} \bar{V}
$$

we obtain the following expressions for entropy $S$ and an expectation value $E$ of the operator $\bar{E}_{I} \equiv \sum_{n} E_{n}|n\rangle_{I} \cdot{ }_{I}\langle n|$ :

$$
S=-\sum_{n} \bar{p}_{n} \ln \bar{p}_{n},
$$

$$
E=\sum_{n, m} E_{n} \bar{p}_{m}\left|V_{n m}\right|^{2},
$$

where $\bar{p}_{n}$ is the diagonal elements of $\bar{P}$. The constraints $\operatorname{Tr} \bar{\rho}=1$ and $\bar{V}^{\dagger} \bar{V}=\mathbf{1}$ are restated as

$$
\begin{gathered}
\sum_{n} \bar{p}_{n}=1, \\
\sum_{l} \bar{V}_{l n}^{*} \bar{V}_{l m}=\delta_{n m} .
\end{gathered}
$$

From these and those expressions, the following correspondence is easily seen:

$$
\begin{aligned}
& S_{\text {ent }} \leftrightarrow S, \\
& E_{\text {ent }} \leftrightarrow E, \\
& C^{\dagger} C \leftrightarrow \widetilde{P} .
\end{aligned}
$$

Hence, a maximum of $S$ in the space of statistical states with a fixed value of $E$ gives a set of maxima of $S_{\text {ent }}$ in a space of quantum states with a fixed value of $E_{\text {ent }}$. (All of them are related by unitary transformations in the subspace $\mathcal{F}_{I I}$.) Thus, since the thermal state $\widetilde{P}_{n m}=e^{-E_{n} / T} \delta_{n m}$ is a maximum of $S$ in a space of statistical states with a fixed value of $E, \quad C_{n m}=e^{-E_{n} / 2 T} \delta_{n m}$ is a maximum of $S_{e n t}$ in a space of pure quantum states with a fixed value of $E_{\text {ent }}$. Here the temperature $T$ should be determined so that $E$ (or $E_{\text {ent }}$ ) has a fixed value. This completes the proof of the general statement.

Therefore, for the system of the scalar field, a state of the form (12) is a maximum of the entanglement entropy in a space of pure quantum states satisfying the SBC, provided that the constant $T$ is determined so that the SBC is satisfied. The value of $T$ is easily determined as $T=T_{H}$ by using the well-known fact that the negative divergence in the Boulware energy density can be canceled by thermal excitations if and only if temperature with respect to the time $t$ is equal to the Hawking temperature. ${ }^{3}$

Finally, by taking the limit $\alpha \rightarrow 0$, we obtain the statement that the Hartle-Hawking state is a maximum of entanglement entropy in a space of pure quantum states satisfying the SBC. ${ }^{4}$ In other words, the Hartle-Hawking state is a curvedspace analogue of the Einstein-Podolsky-Rosen (EPR) state, which is also a maximum of entanglement entropy [5].

From this result we can say that the brick wall model of ' $t$ Hooft [10] seeks the maximal value of entanglement entropy [15]. Thus, the maximal entanglement entropy is equal to the black hole entropy if the cutoff length $\alpha$ is set to be of the order of the Planck length. Although in our arguments we

\footnotetext{
${ }^{3}$ See e.g., Ref. [7].

${ }^{4}$ The Hartle-Hawking state is actually of the form (12) with $T$ $=T_{H}[14]$, provided that $\alpha \rightarrow 0$.
} 
have taken the limit $\alpha \rightarrow 0$, it will be valuable to investigate possibilities that quantum fluctuations of geometries may prevent $\alpha$ from being zero and that the fluctuations of the horizon may be effectively represented as a Planck-order value of $\alpha$. Note that the effect of a non-zero value of $\alpha$ on our arguments should be small enough if the mass of a background black hole is sufficiently large in Planck units.

Our arguments suggests a strong connection among three kinds of thermodynamics: black hole thermodynamics, statistical thermodynamics, and entanglement thermodynamics
$[5,8,9,16]$. Moreover, from the interpretation of entanglement entropy proposed in Ref. [5], it is expected that the Hartle-Hawking state may play an important role in transmitting information by using quantum entanglement to restore temporarily missing information. It will be interesting to investigate such a possibility in detail.

The author would like to thank Professor H. Kodama and Professor W. Israel for their continuing encouragement. This work was supported partially by a Grant-in-Aid for Scientific Research Fund (No. 9809228).
[1] L. Bombelli, R. K. Koul, J. Lee, and R. D. Sorkin, Phys. Rev. D 34, 373 (1986).

[2] M. Srednicki, Phys. Rev. Lett. 71, 666 (1993).

[3] J. D. Bekenstein, Phys. Rev. D 7, 949 (1973).

[4] S. W. Hawking, Commun. Math. Phys. 43, 199 (1975).

[5] S. Mukohyama, Phys. Rev. D 58, 104023 (1998).

[6] J. B. Hartle and S. W. Hawking, Phys. Rev. D 13, 2188 (1976).

[7] S. Mukohyama and W. Israel, Phys. Rev. D 58, 104005 (1998).

[8] S. Mukohyama, M. Seriu, and H. Kodama, Phys. Rev. D 58, 064001 (1998).

[9] S. Mukohyama, Ph. D. thesis, gr-qc/9812079.
[10] G. 't Hooft, Nucl. Phys. B256, 727 (1985).

[11] F. Belgiorno and S. Liberati, Phys. Rev. D 53, 3172 (1996); S. Liberati, Nuovo Cimento Soc. Ital. Fis., B 112, 405 (1997).

[12] F. Belgiorno and M. Martellini, Phys. Rev. D 53, 7073 (1996).

[13] E. T. Jaynes: Papers on Probability, Statistics and Statistical Physics, edited by R. D. Rosenkrantz (Reidel, Boston, 1983).

[14] W. Israel, Phys. Lett. 57A, 107 (1976).

[15] S. Mukohyama, in the proceedings of the the 8th Canadian Conference on General Relativity and Relativistic Astrophysics, gr-qc/9907090.

[16] S. Mukohyama, M. Seriu, and H. Kodama, Phys. Rev. D 55, 7666 (1997). 